\title{
Recurrent Neural Network Interaction Quality Estimation
}

\author{
Louisa Pragst ${ }^{1}$, Stefan Ultes ${ }^{2}$, and Wolfgang Minker ${ }^{1}$ \\ 1 Institute of Communications Engineering, Ulm University, Germany \\ \{louisa.pragst, wolfgang.minker\}@uni-ulm.de \\ 2 Cambridge University, Engineering Department, UK \\ su259@cam.ac.uk
}

\begin{abstract}
Getting a good estimation of the Interaction Quality (IQ) of a spoken dialogue helps to increase the user satisfaction as the dialogue strategy may be adapted accordingly. Therefore, some research has already been conducted in order to automatically estimate the Interaction Quality. This paper adds to this by describing how Recurrent Neural Networks may be used to estimate the Interaction Quality for each dialogue turn and by evaluating their performance on this task. Here, we will show that RNNs may outperform non-recurrent neural networks.
\end{abstract}

Keywords: RNN, Sequential Data, Quality of Dialogue, recurrent neural network, neural network, interaction quality, user satisfaction, spoken dialogue system

\section{Introduction}

While spoken human-computer interaction (HCI) is now wildly available on mobile technical devices, still, the applications are limited and the system behaves in a non-user-adaptive manner, i.e., the system does not take into account shortterm properties of the user. However, having information about these short-term properties or user states like user satisfaction will help rendering this type of HCI more natural and user friendly [1-4].

Automatically deriving the user satisfaction during the ongoing interaction for each turn is a difficult task. Here, we have proposed several approaches for solving this problem before using either static methods [5-7] or sequential methods $[8,9]$. For the latter, the problem is regarded as a sequence of user satisfaction values which are not independent from each other (which seems to be natural as a dialogue interaction may also be considered to be a sequence).

For such sequential problems, recurrent neural networks (RNNs) [10] have shown to perform well for modelling specific problems of Spoken Dialogue Systems (SDSs) [11-14]. An RNN extends a regular neural network by adding cyclic connections with a time delay thus making them very suitable for their application for sequential problems such as the automatic estimation of the user satisfaction which will be represented by the Interaction Quality (IQ) in this work. Hence, in this work, we will investigate and analyse the potential of RNNs 
for estimating the IQ level which will be presented in Sect. 2 together with other relevant related work. For applying RNNs for IQ estimation, we will propose possible approaches with different topologies (Sect. 3). Based on a data set of annotated dialogues, all approaches will be evaluated and their results presented in Sect. 4.

\section{Significant Related Work}

Famous work on determining the satisfaction level automatically is the PARADISE framework by Walker et al. [15]. Assuming a linear dependency between objective measures and User Satisfaction (US), a linear regression model is applied to determine US on the dialogue level. This is not only very costly, as dialogues must be performed with real users, but also inadequate if quality on a finer level is of interest, e.g., on the exchange level. In the following, we will present work on automatically determining the user satisfaction on the exchange level followed by a description of the Interaction Quality and related estimation approaches.

\subsection{User Satisfaction}

For predicting subjective quality measures on the exchange level, several research work has been conducted hitherto. However, most of this body of work lacks of either taking account of the sequential structure of the dialogue or resulting in insufficient performance.

Engelbrecht et al. [16] presented an approach using Hidden Markov Models (HMMs) to model the SDS as a process evolving over time. Performance ratings on a 5 point scale ("bad", "poor", "fair", "good", "excellent") have been applied by the users of the SDS during the dialogue. The interaction was halted while the user rated.A HMM was created consisting of 5 states (one for each rating) and a 6 -dimensional input vector. While Engelbrecht et al. [16] relied on only 6 input variables, we will pursue an approach with 29 input variables. Moreover, we will investigate dialogues of a real world dialogue system annotated with quality labels by expert annotators.

Higashinaka et al. [17] proposed a model for predicting turn-wise ratings for human-human dialogues. Ratings ranging from 1 to 7 were applied by two expert annotators labeling for smoothness, closeness, and willingness. They achieved an $\mathrm{UAR}^{3}$ of only $0.2-0.24$ which is only slightly above the random baseline of 0.14 .

Hara et al. [18] derived turn level ratings from overall ratings of the dialogue which were applied by the users after the interaction on a five point scale within an online questionnaire. Using n-grams to model the dialogue by calculating ngram occurrence frequencies for each satisfaction value showed that results for distinguishing between six classes at any point in the dialogue to be hardly above chance.

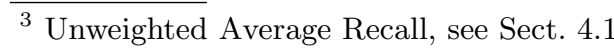




\subsection{The Interaction Quality Paradigm and the LEGO Corpus}

As a more objective means of describing the user's satisfaction with the interaction, Schmitt and Ultes [19] proposed a measure called "Interaction Quality" (IQ) which fulfills the requirements of a quality metric for adaptive dialogue identified by Ultes et al. [20]. For the authors, the main aspect of user satisfaction is that it is assigned by real users. However, this is impractical in many real world scenarios. Therefore, the usage of expert raters is proposed. Further studies have also shown that ratings applied by experts and users have a high correlation [21].

The general idea of the Interaction Quality (IQ) paradigm - IQ being defined as user satisfaction annotated by expert raters - is to derive a number of interaction parameters from the dialogue system and use those as input variables to train a statistical classifier targeting IQ. Interaction quality is modelled on a scale from 5 to 1 representing the ratings "satisfied" (5), "slightly unsatisfied" (4), "unsatisfied" (3), "strongly unsatisfied" (2), and "extremely unsatisfied" (1).

The IQ paradigm [22] is based on automatically deriving interaction parameters from the SDS. These parameters are fed into a statistical classification module which estimates the IQ level of the ongoing interaction at the current system-user-exchange ${ }^{4}$. The interaction parameters are rendered on three levels (see Fig. 1): the exchange level, the window level, and the dialogue level. The exchange level comprises parameters derived from SDS modules Automatic Speech Recognition (ASR), Spoken Language Understanding (SLU), and Dialogue Management (DM) directly. Parameters on the window and the dialogue level are sums, means, frequencies or counts of exchange level parameters. While dialogue level parameters are computed out of all exchanges of the dialogue up to the current exchange, window level parameters are only computed out of the last three exchanges.

These interaction parameters are used as input variables to a statistical classification module with the goal of estimating the IQ value. The statistical model is trained based on annotated dialogues of the Lets Go Bus Information System in Pittsburgh, USA [23]. For the LEGO corpus [24], 200 calls from 2006 consisting of 4,885 exchanges have been annotated by three different raters resulting in a rating agreement of $\kappa=0.54^{5}$. Furthermore, the raters had to follow labeling guidelines to enable a consistent labeling process [24]. The median of all three ratings is used to establish a ground truth for the IQ value of a given turn.

Schmitt et al. [19], [22] performed IQ recognition on the LEGO corpus using linear support vector machines (SVMs). They achieved an $\mathrm{UAR}^{3}$ of 0.58 based on 10 -fold cross-validation which is clearly above the random baseline of 0.2 . Using the same data, Ultes et al. [8] put an emphasis on the sequential character of the IQ measure by applying Hidden Markov Models (HMMs) and Conditioned Hidden Markov Models (CHMMs). Both have been applied using 6-fold cross validation and a reduced feature set of the LEGO corpus achieving an $\mathrm{UAR}^{3}$ of

\footnotetext{
${ }^{4}$ A system-user exchange comprises a system turn followed by a user turn.

${ }^{5} \mathrm{UAR}, \kappa$ and $\rho$ are defined in Sect. 4.1
} 
0.44 for HMMs and 0.39 for CHMMs. In addition to modelling the observation probabilities of the HMM with generic Gaussian Mixture Models [8], Ultes et al. also proposed the combination of static classifiers with HMMs resulting in a hybrid approach [9]: there, the observation probabilities are modelled using the confidence scores of the static classification algorithms. By that, they were able to beat the performance of the confidence-providing SVM by $2.2 \%$ in UAR.

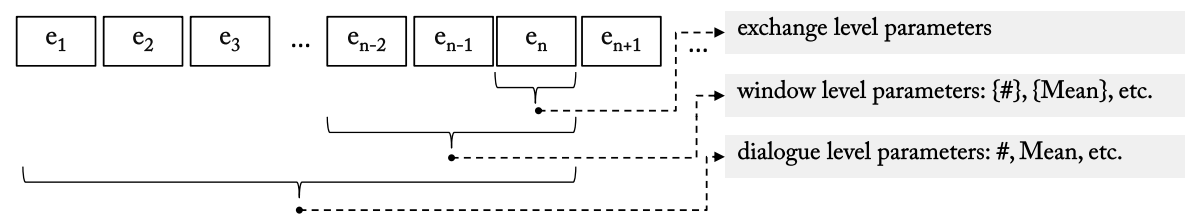

Fig. 1: This figure originally published by Schmitt et al. [24] shows the three parameter levels constituting the interaction parameters: the exchange level containing information about the current exchange, the window level, containing information about the last three exchanges, and the dialogue level containing information about the complete dialogue up to the current exchange.

\section{Recurrent Neural Networks for IQ Estimation}

Following the approaches presented by Ultes et al. [8], we focus on the sequential character of the IQ measure and propose recurrent neural networks [10] for IQ estimation. Recurrent neural networks enhance regular neural networks with cyclic, time delayed connections, that can be used as memory for earlier events. This makes them a reasonable choice for sequential problems such as the one presented.

The architecture of recurrent neural network can be varied by many factors, e.g. the number of layers, the number of nodes in a layer as well as the number, placement and time delay of the recurrent connections. All of these factors potentially influence the performance of the recurrent neural network.

In this work, we evaluate recurrent architectures that differ in the placement of the recurrent connections, as depicted in Fig. 2, as well as the number of nodes in the hidden layer. More variations, such as the number of hidden layers and different time delays, were assessed, but yielded comparable results and are therefore not discussed further in this work.

We analyse the IQ estimation capability of three recurrent architectures for neural networks. They are introduced in the following.

An Elman network [25] is a simple recurrent network, with a recurrent connection from the hidden layer to itself. The previous results of the hidden layer may be combined with the current input values in order to achieve better a estimate of the IQ value. 


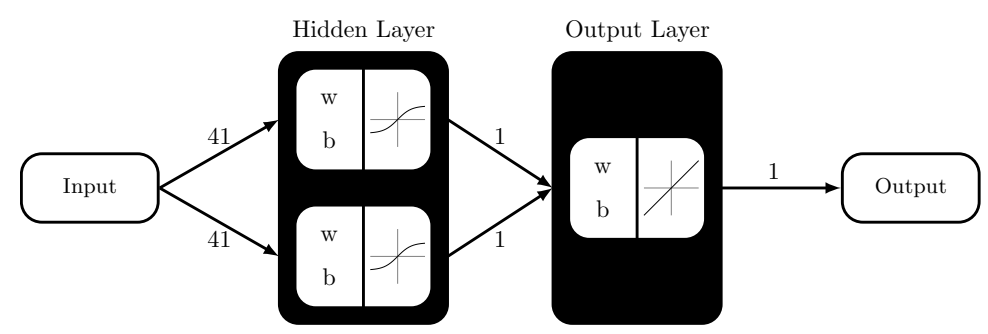

(a) Architecture of a non-recurrent neural network.

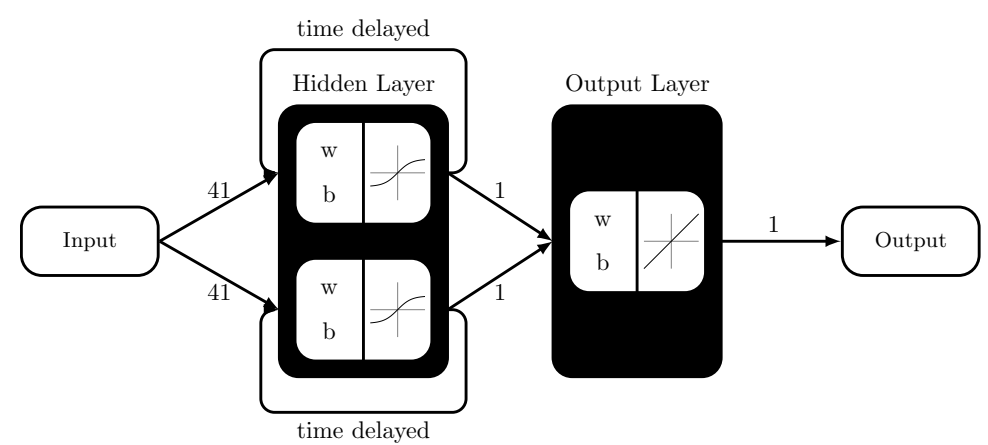

(b) Architecture of an Elman network.

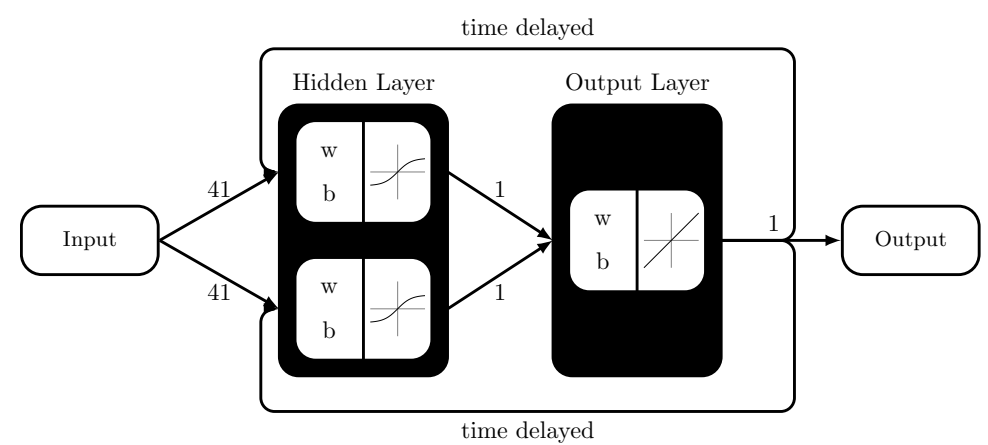

(c) Architecture of a NARX network.

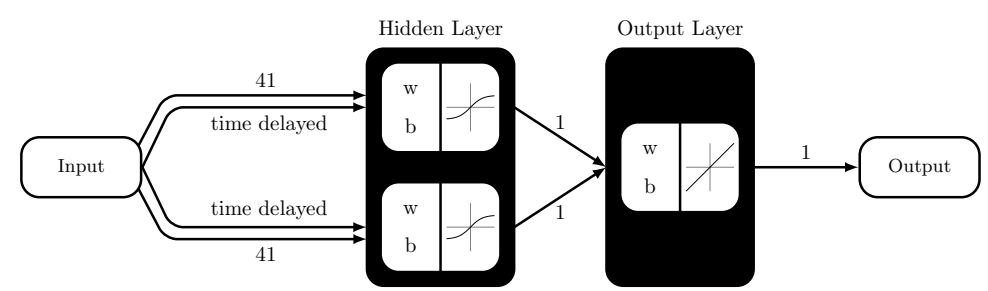

(d) Architecture of a time delay network.

Fig. 2: The different architectures of recurrent neural networks we evaluate in this work, exemplary with 2 nodes in the hidden layer. The nodes of the hidden layer are connected to the input variables and a bias. They use a tanh transfer function. The output layer contains one node that gets it input from the nodes of the previous layer and a bias. It uses a linear transfer function. 
A NARX network [26] utilises a recurrent connection from the output layer to the hidden layer. Thus, the estimated IQ value of the preceding exchange may be used for the current estimation. As raters were instructed to change the IQ value only by one from one exchange to the next (except in severe cases), the estimation might prove to be a valuable input.

Finally, a time delay network [27] is assessed. This architecture incorporates a recurrent connection from the input to the hidden layer. By comparing preceding input values with the current ones, differences that lead to an adjustment of the IQ value might be discovered and taken into consideration.

For all architectures, the nodes of both layers involved in recurrence are interconnected with each other by recurrent connections, and the time delay is set to one time step.

The number of nodes in the hidden layer varies from 2 to 20 . The number of nodes available has an impact on the complexity of the calculation the network is able to perform. A high amount of nodes is beneficial if IQ estimation from the provided features requires an intricate mathematical formula. On the other hand, a high amount of nodes impedes the training process, and a satisfactory result might not be reached with the given amount of training data. By varying the number of nodes, the best trade-off can be determined.

The following characteristics are shared by all networks covered in this work. The networks contain only one hidden layer. All nodes of this layer are connected to all input variables as well as a bias and use a tanh transfer function. The output layer consists of one node, that gets its input from all nodes of the hidden layer and possibly a recurrent connection. It has a bias and uses a linear transfer function.

The networks perform a regression by implementing this architecture in their output layer, although the presented problem is a classification task. A different output layer implementing classification was tested and yielded worse results. Therefore, this architecture was adopted. The final estimated IQ value is obtained by rounding the result of the network.

\section{Experiments and Results}

The performance of different recurrent neural networks is evaluated with the metrics described in the following.

\subsection{Evaluation Metrics}

Interaction Quality estimation is done by using three commonly used evaluation metrics: Unweighted Average Recall (UAR), Cohen's Kappa [28] and Spearman's $R h o$ [29]. These are also selected as the same metrics have been used in Schmitt et al. [22] as well.

Recall in general is defined as the rate of correctly classified samples belonging to one class. The recall in UAR for multi-class classification problems with $N$ 
classes recall $i$ is computed for each class $i$ and then averaged over all class-wise recalls:

$$
U A R=\frac{1}{N} \sum_{i=1}^{N} \text { recall }_{i} .
$$

Cohen's Kappa measures the relative agreement between two corresponding sets of ratings. In our case, we compute the number of label agreements corrected by the chance level of agreement divided by the maximum proportion of times the labelers could agree. However, Cohen's weighted Kappa is applied as ordinal scores are compared [30]. A weighting factor $w$ is introduced reducing the discount of disagreements the smaller the difference is between two ratings:

$$
w=\frac{\left|r_{1}-r_{2}\right|}{\left|r_{\max }-r_{\min }\right|} .
$$

Here, $r_{1}$ and $r_{2}$ denote the rating pair and $r_{\max }$ and $r_{\min }$ the maximum and minimum ratings possible.

Correlation between two variables describes the degree by which one variable can be expressed by the other. Spearman's Rho is a non-parametric method assuming a monotonic function between the two variables [29].

\section{$4.2 \quad$ Experimental Setup}

To evaluate the performance of recurrent neural networks for IQ estimation, we use the LEGO corpus presented in Sect. 2.2. Exchanges with less than three IQ ratings are excluded from the evaluation for being too unreliable, just as dialogues with less than three exchanges, as the IQ value can not be adjusted before the third exchange. Furthermore, only numeric features which can be automatically derived are used, leaving 41 input features.

For this corpus, we evaluate the performance of the recurrent neural network architectures described in Sect. 3 using 10-fold cross validation, as well as a neural network without recurrence in order to determine the impact of sequential information. Whole dialogues are assigned to the train or test condition of the 10 -fold cross validation, instead of assigning each exchange separately, in order to account for the sequential character of the data. The neural networks are trained with the dialogues of the train condition using dynamic backpropagation [31]. Each dialogue is provided to the neural network sequentially, as in real conversation.

In related work ([8,9], [19], [22]), the evaluation metrics are obtained using the available data in its entirety. As we feel that this approach does not adequately take into account the sequentiality of the data, we utilised a different approach: the evaluation metrics are obtained for each one of the 200 dialogues separately and then averaged over all dialogues. The performance of SVMs [19], [22] has been re-evaluated using this new approach in order to achieve comparability. This results in an UAR of 0.55 , a $\kappa$ of 0.30 , and a $\rho$ of 0.50 for SVMs. 


\subsection{Results}

The average results over all 200 dialogues for all configurations and evaluation metrics can be found in Table 1 .

The best results are achieved using a NARX network with ten nodes in the hidden layer: this architecture yields an UAR of 0.60 , a $\kappa$ of 0.37 , and a $\rho$ of 0.55. It outperforms the SVM approach (UAR: $0.55, \kappa: 0.30, \rho: 0.50$ ).

When comparing the recurrent architectures with the non-recurrent architecture, statistical tests suggest that recurrence does not significantly increase the performance of neural networks for IQ estimation $(p>0.05$ for Elman and NARX network). The time delay network even significantly decreases the performance $(p<0.001)$.

These results might be due to the fact that training recurrent neural networks is more complex than training non-recurrent neural networks [32,33]. By utilising more elaborate training approaches, it may be possible to achieve different results.

Furthermore, the number of nodes does not appear to have a significant impact on the ability of a neural network to estimate the IQ value. While occasional significant differences between consecutive levels of the number of nodes can be found, those differences are isolated and not consistent across evaluation metrics. The assumption that the performance would become better at first as more complex calculations are possible, and then decrease as training becomes more difficult, can not be affirmed.

\section{Conclusion}

This work presented recurrent neural networks for the estimation of Interaction Quality on the exchange level. It could be shown that both non-recurrent and recurrent neural networks can outperform previous approaches such as SVMs in this task.

The sequential structure of dialogues suggests that recurrent neural networks might exceed the performance of non-recurrent neural networks in estimating the Interaction Quality on the exchange level, as they incorporate information about previous exchanges in their calculations. By evaluating the performance of different recurrent neural network architectures and comparing them to the performance of a neural network without recurrence, we found that Elman and NARX networks do not significantly outperform a corresponding non-recurrent neural network. Moreover, the time-delay network performs significantly worse than the non-recurrent network. This might be due to the fact that the training of recurrent networks is more complex than the training of non-recurrent networks. It is probable that more training data or a more refined training approach is needed.

For future research, the number of input features may be reduced in a meaningful way, or a larger corpus of training data may be used, in order to train the recurrent neural networks more efficiently. Furthermore, the training approach 
Table 1: Results given by the Unweighted Average Recall (UAR), Cohen's Kappa $(\kappa)$, and Spearman's Rho $(\rho)$.

(a) Performance of the baseline network.

\begin{tabular}{cccc}
\hline \#nodes & UAR & $\kappa$ & $\rho$ \\
\hline $\mathbf{2}$ & 0.56 & 0.35 & 0.53 \\
$\mathbf{3}$ & 0.55 & 0.34 & 0.52 \\
$\mathbf{4}$ & 0.56 & 0.34 & 0.52 \\
$\mathbf{5}$ & 0.56 & 0.35 & 0.51 \\
$\mathbf{6}$ & 0.55 & 0.35 & 0.53 \\
$\mathbf{7}$ & 0.57 & 0.36 & 0.52 \\
$\mathbf{8}$ & 0.54 & 0.33 & 0.52 \\
$\mathbf{9}$ & 0.56 & 0.35 & 0.52 \\
$\mathbf{1 0}$ & 0.55 & 0.35 & 0.52 \\
$\mathbf{1 1}$ & 0.53 & 0.31 & 0.49 \\
$\mathbf{1 2}$ & 0.52 & 0.34 & $\mathbf{0 . 5 4}$ \\
$\mathbf{1 3}$ & 0.57 & 0.36 & 0.53 \\
$\mathbf{1 4}$ & $\mathbf{0 . 5 8}$ & $\mathbf{0 . 3 8}$ & $\mathbf{0 . 5 4}$ \\
$\mathbf{1 5}$ & 0.56 & 0.35 & 0.52 \\
$\mathbf{1 6}$ & 0.57 & 0.37 & 0.53 \\
$\mathbf{1 7}$ & 0.57 & 0.35 & 0.53 \\
$\mathbf{1 8}$ & 0.57 & 0.37 & 0.53 \\
$\mathbf{1 9}$ & $\mathbf{0 . 5 8}$ & 0.36 & 0.53 \\
$\mathbf{2 0}$ & 0.56 & 0.35 & 0.53 \\
\hline
\end{tabular}

(c) Performance of the NARX network.

\begin{tabular}{cccc}
\hline \#nodes & UAR & $\kappa$ & $\rho$ \\
\hline $\mathbf{2}$ & 0.57 & 0.34 & 0.52 \\
$\mathbf{3}$ & 0.55 & 0.33 & 0.53 \\
$\mathbf{4}$ & 0.57 & 0.35 & 0.52 \\
$\mathbf{5}$ & 0.54 & 0.32 & 0.52 \\
$\mathbf{6}$ & 0.56 & 0.32 & 0.51 \\
$\mathbf{7}$ & 0.55 & 0.33 & 0.52 \\
$\mathbf{8}$ & 0.57 & 0.35 & 0.54 \\
$\mathbf{9}$ & 0.57 & 0.35 & 0.53 \\
$\mathbf{1 0}$ & $\mathbf{0 . 6 0}$ & $\mathbf{0 . 3 7}$ & $\mathbf{0 . 5 5}$ \\
$\mathbf{1 1}$ & 0.58 & 0.36 & 0.53 \\
$\mathbf{1 2}$ & 0.57 & 0.33 & 0.51 \\
$\mathbf{1 3}$ & 0.52 & 0.31 & 0.51 \\
$\mathbf{1 4}$ & 0.58 & 0.35 & 0.52 \\
$\mathbf{1 5}$ & 0.57 & 0.35 & 0.52 \\
$\mathbf{1 6}$ & 0.57 & 0.34 & 0.53 \\
$\mathbf{1 7}$ & 0.54 & 0.31 & 0.49 \\
$\mathbf{1 8}$ & 0.56 & 0.33 & 0.51 \\
$\mathbf{1 9}$ & 0.52 & 0.32 & 0.51 \\
$\mathbf{2 0}$ & 0.58 & 0.35 & 0.52 \\
\hline
\end{tabular}

(b) Performance of the Elman network.

\begin{tabular}{cccc}
\hline \#nodes & UAR & $\kappa$ & $\rho$ \\
\hline $\mathbf{2}$ & 0.54 & 0.31 & 0.48 \\
$\mathbf{3}$ & 0.55 & 0.33 & 0.51 \\
$\mathbf{4}$ & 0.56 & 0.33 & 0.52 \\
$\mathbf{5}$ & 0.56 & 0.33 & 0.50 \\
$\mathbf{6}$ & 0.55 & 0.32 & 0.52 \\
$\mathbf{7}$ & 0.57 & 0.35 & 0.52 \\
$\mathbf{8}$ & 0.55 & 0.34 & 0.51 \\
$\mathbf{9}$ & 0.55 & 0.34 & 0.50 \\
$\mathbf{1 0}$ & 0.56 & 0.34 & 0.52 \\
$\mathbf{1 1}$ & 0.56 & 0.35 & 0.54 \\
$\mathbf{1 2}$ & 0.57 & 0.35 & 0.53 \\
$\mathbf{1 3}$ & 0.57 & 0.35 & 0.52 \\
$\mathbf{1 4}$ & 0.58 & $\mathbf{0 . 3 7}$ & 0.53 \\
$\mathbf{1 5}$ & 0.58 & 0.35 & 0.51 \\
$\mathbf{1 6}$ & 0.55 & 0.35 & 0.53 \\
$\mathbf{1 7}$ & $\mathbf{0 . 5 9}$ & $\mathbf{0 . 3 7}$ & $\mathbf{0 . 5 5}$ \\
$\mathbf{1 8}$ & 0.58 & 0.35 & 0.53 \\
$\mathbf{1 9}$ & 0.57 & 0.36 & 0.53 \\
$\mathbf{2 0}$ & 0.58 & 0.36 & 0.53 \\
\hline
\end{tabular}

(d) Performance of the time delay network.

\begin{tabular}{cccc}
\hline \#nodes & UAR & $\kappa$ & $\rho$ \\
\hline $\mathbf{2}$ & 0.55 & 0.32 & 0.50 \\
$\mathbf{3}$ & 0.55 & $\mathbf{0 . 3 3}$ & $\mathbf{0 . 5 1}$ \\
$\mathbf{4}$ & 0.55 & 0.32 & 0.49 \\
$\mathbf{5}$ & 0.56 & $\mathbf{0 . 3 3}$ & 0.49 \\
$\mathbf{6}$ & 0.51 & 0.30 & 0.49 \\
$\mathbf{7}$ & 0.53 & 0.30 & 0.48 \\
$\mathbf{8}$ & 0.55 & $\mathbf{0 . 3 3}$ & 0.50 \\
$\mathbf{9}$ & 0.52 & 0.29 & 0.47 \\
$\mathbf{1 0}$ & 0.55 & $\mathbf{0 . 3 3}$ & 0.50 \\
$\mathbf{1 1}$ & 0.55 & 0.32 & 0.48 \\
$\mathbf{1 2}$ & 0.55 & 0.32 & 0.49 \\
$\mathbf{1 3}$ & 0.54 & 0.32 & 0.49 \\
$\mathbf{1 4}$ & 0.56 & $\mathbf{0 . 3 3}$ & 0.50 \\
$\mathbf{1 5}$ & $\mathbf{0 . 5 7}$ & 0.31 & 0.48 \\
$\mathbf{1 6}$ & 0.53 & 0.30 & 0.47 \\
$\mathbf{1 7}$ & 0.55 & 0.32 & 0.47 \\
$\mathbf{1 8}$ & 0.52 & 0.29 & 0.46 \\
$\mathbf{1 9}$ & 0.55 & 0.32 & 0.48 \\
$\mathbf{2 0}$ & 0.51 & 0.29 & 0.44 \\
\hline
\end{tabular}


could be refined. Different recurrent architectures, for example a combination of the networks presented in this work, could be tested in addition.

Acknowledgments This paper is part of a project that has received funding from the European Union's Horizon 2020 research and innovation programme under grant agreement No 645012 .

\section{References}

1. Ultes, S., Heinroth, T., Schmitt, A., Minker, W.: A theoretical framework for a usercentered spoken dialog manager. In: Proceedings of the Paralinguistic Information and its Integration in Spoken Dialogue Systems Workshop. pp. 241 - 246. Springer (Sep 2011)

2. Ultes, S., Dikme, H., Minker, W.: Dialogue management for usercentered adaptive dialogue. In: Proceedings of IWSDS (Jan 2014), http://www .uni-ulm.de/fileadmin/website\_uni\_ulm/allgemein/2014 \ _iwsds $\bigwedge-i w s d s 2014 \backslash$ lp\_ultes.pdf

3. Ultes, S., Dikme, H., Minker, W.: First insight into quality-adaptive dialogue. In: LREC. pp. 246-251 (May 2014)

4. Ultes, S., Kraus, M., Schmitt, A., Minker, W.: Quality-adaptive spoken dialogue initiative selection and implications on reward modelling. In: Proceedings of SIGDIAL. pp. 374-383. ACL (Sep 2015)

5. Ultes, S., Minker, W.: Improving interaction quality recognition using error correction. In: Proceedings of SIGDIAL. pp. 122-126. ACL (Aug 2013), http: //www.aclweb.org/anthology/W/W13/W13-4018

6. Ultes, S., Minker, W.: Interaction quality: A review. Bulletin of Siberian State Aerospace University named after academician M.F. Reshetnev (4), 153-156 (2013), http://www.vestnik.sibsau.ru/images/vestnik/ves450.pdf

7. Ultes, S., Platero Sánchez, M.J., Schmitt, A., Minker, W.: Analysis of an extended interaction quality corpus. In: Proceedings of IWSDS (Jan 2015)

8. Ultes, S., ElChabb, R., Minker, W.: Application and evaluation of a conditioned hidden markov model for estimating interaction quality of spoken dialogue systems. In: Proceedings of IWSDS. pp. 141-150. Springer (Nov 2012)

9. Ultes, S., Minker, W.: Interaction quality estimation in spoken dialogue systems using hybrid-hmms. In: Proceedings of SIGDIAL. pp. 208-217. ACL (Jun 2014), http://www . aclweb.org/anthology/W14-4328

10. Hochreiter, S., Schmidhuber, J.: Long short-term memory. Neural computation 9(8), 1735-1780 (1997)

11. Mesnil, G., He, X., Deng, L., Bengio, Y.: Investigation of recurrent-neural-network architectures and learning methods for spoken language understanding. In: INTERSPEECH. pp. 3771-3775 (2013)

12. Henderson, M., Thomson, B., Young, S.: Robust dialog state tracking using delexicalised recurrent neural networks and unsupervised adaptation. In: SLT. pp. 360 365. IEEE (2014)

13. Wen, T.H., Gačić, M., Kim, D., Mrkšic, N., Su, P.H., Vandyke, D., Young, S.: Stochastic language generation in dialogue using recurrent neural networks with convolutional sentence reranking. pp. 275-284. ACL (Sep 2015) 
14. Su, P.H., Vandyke, D., Gasic, M., Kim, D., Mrksic, N., Wen, T.H., Young, S.: Learning from real users: Rating dialogue success with neural networks for reinforcement learning in spoken dialogue systems. arXiv preprint arXiv:1508.03386 (2015)

15. Walker, M., Litman, D.J., Kamm, C.A., Abella, A.: Paradise: a framework for evaluating spoken dialogue agents. In: Proceedings of ACL. pp. 271-280. ACL, Morristown, NJ, USA (1997)

16. Engelbrecht, K.P., Gödde, F., Hartard, F., Ketabdar, H., Möller, S.: Modeling user satisfaction with hidden markov model. In: Proceedings of SIGDIAL. pp. 170-177. ACL, Morristown, NJ, USA (2009)

17. Higashinaka, R., Minami, Y., Dohsaka, K., Meguro, T.: Issues in predicting user satisfaction transitions in dialogues: Individual differences, evaluation criteria, and prediction models. In: Spoken Dialogue Systems for Ambient Environments, Lecture Notes in Computer Science, vol. 6392, pp. 48-60. Springer Berlin / Heidelberg (2010), 10.1007/978-3-642-16202-2_5

18. Hara, S., Kitaoka, N., Takeda, K.: Estimation method of user satisfaction using n-gram-based dialog history model for spoken dialog system. In: Proceedings of LREC. ELRA, Valletta, Malta (May 2010)

19. Schmitt, A., Ultes, S.: Interaction quality: Assessing the quality of ongoing spoken dialog interaction by experts - and how it relates to user satisfaction. Speech Communication (2015), accepted for publication

20. Ultes, S., Schmitt, A., Minker, W.: Towards quality-adaptive spoken dialogue management. In: NAACL-HLT: SDCTD. pp. 49-52. ACL, Montréal, Canada (Jun 2012), http://www.aclweb.org/anthology/W12-1819

21. Ultes, S., Schmitt, A., Minker, W.: On quality ratings for spoken dialogue systems - experts vs. users. In: Proceedings of NAACL-HLT. pp. 569-578. ACL (Jun 2013)

22. Schmitt, A., Schatz, B., Minker, W.: Modeling and predicting quality in spoken human-computer interaction. In: Proceedings of SIGDIAL. pp. 173-184. ACL, Portland, Oregon, USA (Jun 2011)

23. Raux, A., Bohus, D., Langner, B., Black, A.W., Eskenazi, M.: Doing research on a deployed spoken dialogue system: One year of let's go! experience. In: Proc. of ICSLP (Sep 2006)

24. Schmitt, A., Ultes, S., Minker, W.: A parameterized and annotated spoken dialog corpus of the cmu let's go bus information system. In: LREC. pp. 3369-337 (May 2012)

25. Elman, J.L.: Finding structure in time. Cognitive science 14(2), 179-211 (1990)

26. Lin, T., Horne, B.G., Tiňo, P., Giles, C.L.: Learning long-term dependencies in narx recurrent neural networks. Neural Networks, IEEE Transactions on 7(6), 1329-1338 (1996)

27. Waibel, A., Hanazawa, T., Hinton, G., Shikano, K., Lang, K.J.: Phoneme recognition using time-delay neural networks. Acoustics, Speech and Signal Processing, IEEE Transactions on 37(3), 328-339 (1989)

28. Cohen, J.: A coefficient of agreement for nominal scales. In: Educational and Psychological Measurement. vol. 20, pp. 37-46 (Apr 1960)

29. Spearman, C.E.: The proof and measurement of association between two things. American Journal of Psychology 15, 88-103 (1904)

30. Cohen, J.: Weighted kappa: Nominal scale agreement provision for scaled disagreement or partial credit. Psychological bulletin 70(4), 213 (1968)

31. De Jesús, O., Hagan, M.T.: Backpropagation algorithms for a broad class of dynamic networks. Neural Networks, IEEE Transactions on 18(1), 14-27 (2007) 
32. De Jesús, O., Horn, J.M., Hagan, M.T.: Analysis of recurrent network training and suggestions for improvements. In: Neural Networks, 2001. Proceedings. IJCNN'01. International Joint Conference on. vol. 4, pp. 2632-2637. IEEE (2001)

33. Horn, J., De Jesús, O., Hagan, M.T.: Spurious valleys in the error surface of recurrent networksanalysis and avoidance. Neural Networks, IEEE Transactions on 20(4), 686-700 (2009) 